Unfallchirurg 2021 · 124:685-686

https://doi.org/10.1007/s00113-021-01043-1

Angenommen: 14. Juni 2021

(c) Springer Medizin Verlag $\mathrm{GmbH}$, ein Teil von Springer Nature 2021

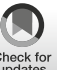

\section{Autor}
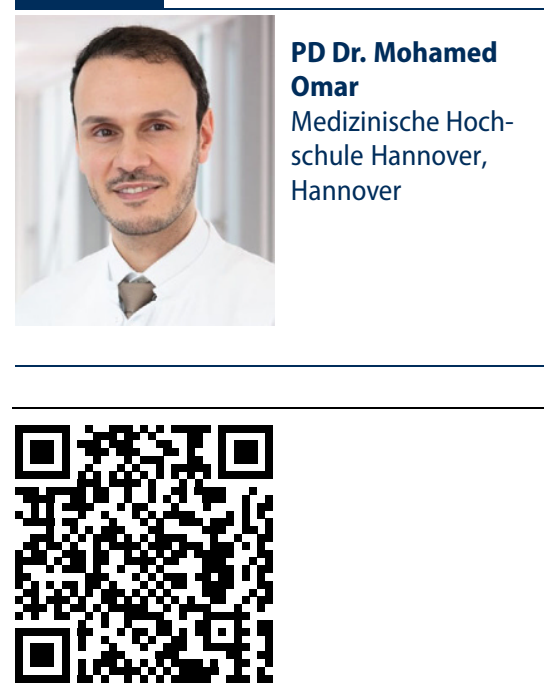

QR-Code scannen \& Beitrag online lesen

\title{
Tumorbedingte pathologische Frakturen
}

\author{
Mohamed Omar ${ }^{1,2,3} \cdot$ P. Ivanyi ${ }^{2,3,4,5}$ \\ ${ }^{1}$ Klinik für Unfallchirurgie, Medizinische Hochschule Hannover, Hannover, Deutschland \\ ${ }^{2}$ Sarkomzentrum Hannover, Medizinische Hochschule Hannover, Hannover, Deutschland \\ ${ }^{3}$ Comprehensive Cancer Center-Niedersachsen (CCC-N), Hannover, Deutschland \\ ${ }^{4}$ Klinik für Hämatologie, Hämostaseologie, Onkologie und Stammzelltransplantation, Medizinische \\ Hochschule Hannover, Hannover, Deutschland \\ ${ }^{5}$ Interdisziplinäre Immun-Onkologische Arbeitsgruppe (ICOG) des CCC-N, Hannover, Deutschland
}

Liebe Leserinnen und Leser,

tumorbedingte pathologische Frakturen begegnen den Unfallchirurgen im Laufe des Arbeitslebens wiederkehrend. Anders als bei der allgemeinen Traumaversorgung ist hierbei nicht nur die schneidende Profession gefragt, sondern auch eine Vielzahl anderer Fachdisziplinen in die Therapie eingebunden. Nur ein enges interdisziplinäres Zusammenspiel, in dem die verschiedenen Akteure die Entscheidungsgrundlagen und geplanten Maßnahmen der beteiligten Fachdisziplinen kennen und verstehen, bildet die Grundlage dafür, ein bestmögliches Ergebnis für den Patienten erzielen zu können. In diesem Sinne besitzt dieses Leitthema nicht nur einen chirurgischen, sondern auch einen internistisch-onkologischen Herausgeber. Auch in der Auswahl der Artikel und der Autoren findet sich ein breites Spektrum von Experten aus den Bereichen Unfallchirurgie, Orthopädie, Onkologie, Radiologie, Radioonkologie und Pathologie. Zuletzt - auch wenn sie keinen eigenen Beitrag im Rahmen dieser Ausgabe leisten - verdienen auch die onkologisch verantwortlichen Kollegen der jeweiligen Organsysteme aus der Hämatologie, Gynäkologie, Urologie, Dermatologie, Pneumologie und Gastroenterologie sowie Nuklearmediziner und Humangenetiker Erwähnung, da sie einen entscheidenden Beitrag zum Gesamtkonzept der Behandlung liefern.

Obwohl pathologische Frakturen unter allen Verletzungen insgesamt nur einen geringen Anteil ausmachen, ist eine Aus- einandersetzung mit diesem Thema unabdingbar, da ein Übersehen oder die Einleitung einer inadäquaten Therapie erhebliche Konsequenzen für den Patienten nach sich ziehen kann. Der chirurgische Reflex, eine sofortige Therapie einzuleiten, muss in vielen Fällen zunächst zurückgestellt werden, zugunsten der Erarbeitung eines onkologischen Gesamtkonzepts. Die Versorgungsstrategien und Therapieempfehlung haben sich über die letzten Jahrzehnte erheblich verändert - und dies ist nicht alleine auf moderne chirurgische Verfahren zurückzuführen. Im Zuge innovativer onkologischer Therapien steigen die Lebenserwartung und damit die Wahrscheinlichkeit des Auftretens einer pathologischen Fraktur. Ebenso überleben Patienten mit skeletaler Metastasierung heutzutage deutlich länger, sodass ein prognosestratifiziertes Vorgehen erforderlich wird. Auch wenn das Vorliegen einer pathologischen Fraktur auf der Grundlage einer Metastasierung formal eine palliative Situation darstellt, ist dies nicht gleichbedeutend mit einer schlechten Prognose. In vielen Fällen sind mehrjährige Verläufe zu erwarten, und $u$. U. ist ein kuratives Vorgehen anzustreben. Die Behandlung steht also nicht immer im Zeichen der Palliation, sondern kann prognostisch relevant werden.

\section{I) Bei tumorbedingten pathologischen Frakturen ist ein enges interdisziplinäres Zusammenspiel essenziell}

Einer der Herausforderungen bei der Behandlung von pathologischen Frakturen 


\section{Einführung zum Thema}

besteht darin, dass zum Zeitpunkt der Diagnosestellung mitunter das Vorliegen eines Tumorleiden nicht bekannt ist und die Fraktur die Erstmanifestation der zugrunde liegenden Erkrankung darstellt. Entsprechend erfolgt der medizinische Erstkontakt nicht zwangsläufig in einem onkologisch spezialisierten Zentrum. Umso wichtiger erscheinen in diesem Zusammenhang Kenntnisse von diagnostischen und strategischen Aspekten in der grundsätzlichen Herangehensweise. In diesem Leitthema soll daher u. a. die Fähigkeit vermittelt werden, eine Stratifizierung der Patienten in Bezug auf die Erforderlichkeit einer Behandlung in einem spezialisierten Zentrum vornehmen zu können. Ferner sollen diagnostische, chirurgische und onkologische Aspekte erörtert werden, die als Handwerkszeug dienen sollen, sich der Problematik anzunähern. Aspekte, die uns besonders wichtig erschienen, werden in redundanter Weise dargestellt, um ihre Relevanz zu unterstreichen.

Wir wünschen, Ihnen mit diesem Beitrag einen lehrreichen und praktisch orientierten Leitfaden zu liefern, der Sie in Ihrer alltäglichen Arbeit unterstützt.

Mohamed Omar und Philipp Ivanyi

\section{Korrespondenzadresse}

\section{PD Dr. Mohamed Omar}

Klinik für Unfallchirurgie, Medizinische Hochschule Hannover

Carl-Neuberg-Straße 1, 30625 Hannover, Deutschland

omar.mohamed@mh-hanover.de

Interessenkonflikt. M. Omar und P. Ivanyi geben an, dass kein Interessenkonflikt besteht.

In eigener Sache

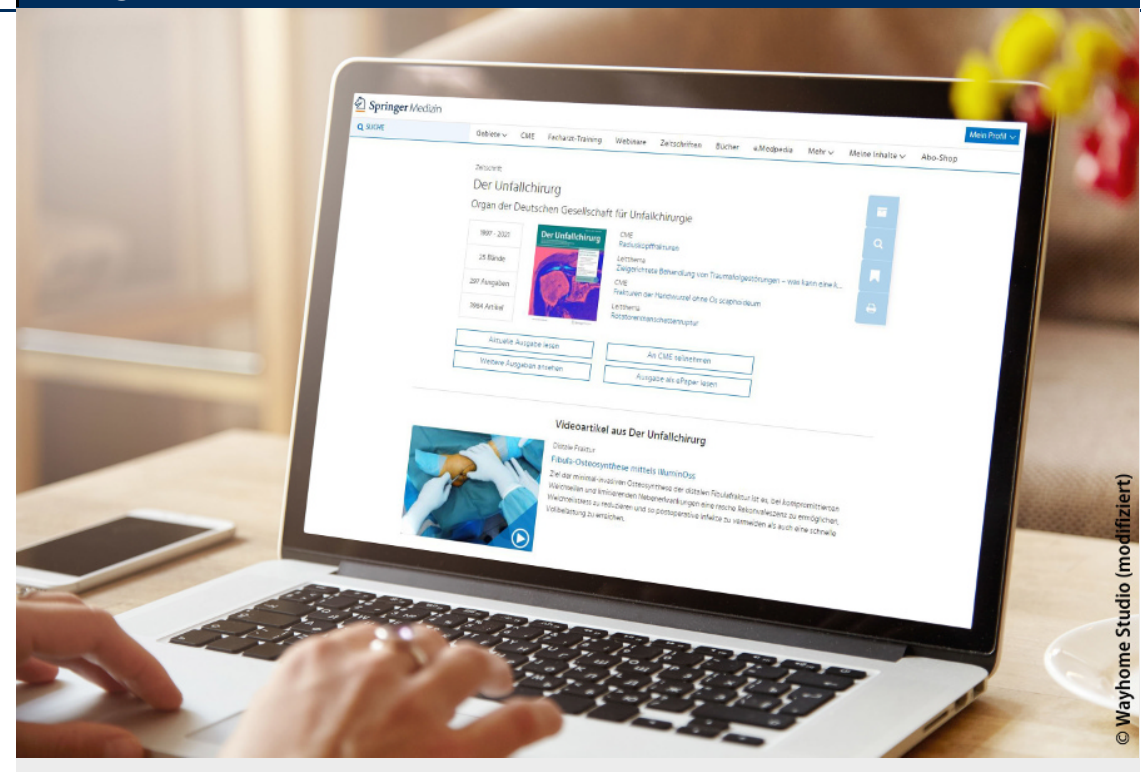

\section{Auch online Zugang zu allen Beiträgen von Der Unfallchirurg}

\section{Wussten Sie, dass Sie als Abonnent*in dieser Zeitschrift automatisch online} Zugriffsrechte auf das gesamte Beitragsarchiv haben?

Durch Ihr Abonnement von Der Unfallchirurg erhalten Sie jeden Monat die aktuelle Ausgabe der Zeitschrift nach Hause geliefert. Doch damit nicht genug: Sie haben mit dem Abonnement außerdem Zugriff auf das gesamte Online-Archiv Ihrer Zeitschrift.

Und so einfach geht es:

Registrieren Sie sich einmal über www.springermedizin.de/register:

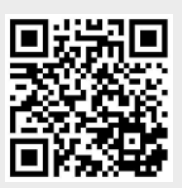

Über diesen QR-Code schnell und einfach registrieren

Bei der Registrierung geben Sie einfach Ihren Vor- und Nachname und Lieferadresse wie beim Abonnement der Zeitschrift (siehe Adressaufkleber auf Ihrem Heft) an. So kann im System die Zugehörigkeit zu Ihrer Zeitschrift sichergestellt werden.

Sollten Fragen oder Probleme auftauchen, wenden Sie sich einfach an den Kundenservice:

kundenservice@springermedizin.de
Aufgrund des Heilmittelwerbegesetzes dürfen die Inhalte der Website nur medizinischen Fachkreisen zur Verfügung gestellt werden. Bei der Anmeldung bitten wir Sie deshalb einen Berufsnachweis vorzulegen. Bei Mediziner*innen mit Mitgliedschaft in der deutschen Ärztekammer reicht die einheitliche Fortbildungsnummer (EFN). Als Angehörige ${ }^{*}$ r eines medizinischen Berufs schicken Sie eine Bestätigung der Arbeitsstelle. Sind Sie Student*in, dann senden Sie bitte den Studiennachweis mit Angabe des Studiengangs ganz unkompliziert an: kundenservice@springermedizin.de.

Mit Benutzername und Passwort haben Sie außerdem Zugang zu den freien Inhalten auf den Seiten von: https://www.springermedizin.de/ https://www.aerztezeitung.de/ 